\title{
Chloromethylated Poly(naphthyl methacrylate) as Electron Beam and Photo Resist
}

\author{
Saburo ImAmura, Toshiaki TAmamura, and Osamu Kogure \\ Polymer Section, Ibaraki Electrical Communication Laboratory, \\ Nippon Telegraph and Telephone Public Corporation, \\ Tokai, Ibaraki 319-11, Japan \\ (Received February 3, 1984)
}

\begin{abstract}
Resists applicable to both electron beam and mid UV ( $300-400 \mathrm{~nm})$ photolithographies were designed, and chloromethylated poly(naphthyl methacrylates) (CMN) were synthesized. The sensitivity of CMN with $M_{w}$ of $1.4 \times 10^{5}$, which resolved $0.5 \mu \mathrm{m}$ of line and space was $4.5 \mu \mathrm{Ccm}^{-2}$. CMN could be used as photo-resist by the addition of suitable photo-sensitizer such as Michler's ketone. CMN with $3 \mathrm{wt} \%$ Michler's ketone showed high sensitivity $\left(D_{0.5}=\right.$ $100 \mathrm{~mJ} \mathrm{~cm}^{-2}$ ) and resolved $2 \mu \mathrm{m}$ minimum line.

KEY WORDS Resist / Electron Beam Resist / Photo Resist / Aromatic

Polymer / Poly(naphthyl methacrylate) / Dry-Etching / Lithography /

Chloromethylated Poly(naphthyl methacrylate) /
\end{abstract}

It is well known that the resolution of photolithography is limited by diffraction of light. Decreasing wavelength of exposure source could improve the resolution. A higher resolution is actually obtained in deep UV lithography using wavelength less than $300 \mathrm{~nm}$. However, mirror projection system must be employed in deep UV lithography, because of difficulty in producing lenses for this wavelength region. By contrast, step-andrepeat system with refractive optics, which is now regarded as the most promising system for VLSI fabrication with a minimum pattern size less than $2.0 \mu \mathrm{m}$, normally uses a monochromatic light of $436 \mathrm{~nm}$ from a high pressure mercury lamp. A practical submicron photolithography is expected by applying 365 or $310 \mathrm{~nm}$ wavelength in step-and-repeat system, and the development of both lens system and resist materials is required for this mid-UV region.

The present approach to mid-UV resists is the modification of conventional photoresists. ${ }^{1}$ However, we have developed several high re- solution resists for X-ray and electron beam lithography, ${ }^{2-4}$ and the sensitization of these resists to mid-UV light will be worthy to try. This trial could open the possibility of electron beam-photo hybrid lithography ${ }^{5,6}$ where relatively large patterns were printed with UV light and very fine patterns were delineated with a finely focussed electron beam in the same resist. There seems to be no conventional photoresist possesing practical resolution and sensitivity to electron beam.

This paper deals with new negative type resist for both electron beam and mid-UV lithographies.

\section{MOLECULAR DESIGN}

Another important requirement for resists in the latest VLSI fabrication processes is high dry-etching durability. Aromatic ring structure is known to add dry-etching durability to resists, because of relative stability of benzene ring against various dry-etching reactions. ${ }^{2,7}$ Chloromethylated polystyrene (CMS) is one of 
the representative negative resists for dryetching processes. ${ }^{2}$ However, CMS and other resists containing benzene rings have no characteristic absorption in a wavelength region longer than $300 \mathrm{~nm}$.

Since it is known that polyaromatic ring structures have strong $\pi-\pi^{*}$ absorption in $300-400 \mathrm{~nm}$, a high sensitivity to mid-UV light could be obtained by polyaromatic polymers with or without suitable sensitizer. These polymers will show a higher dry-etching durability and a high glass-transition temperature $\left(T_{\mathrm{g}}\right)$ which is necessary to suppress the swelling of resist polymers during development. However three disadvantages are expected in polyaromatic polymers, that is, lower sensitivity because of stabilized ring structure, poor solubility, and difficulty in synthesizing resist polymers. In order to make these effects clear, a series of polyaromatic polymers of which structures are shown in Figure 1 were synthesized. As a main chain, methacrylic polymer structure was chosen because many methacrylic polymers such as poly(methyl methacrylate) and poly(glycidyl methacrylate),

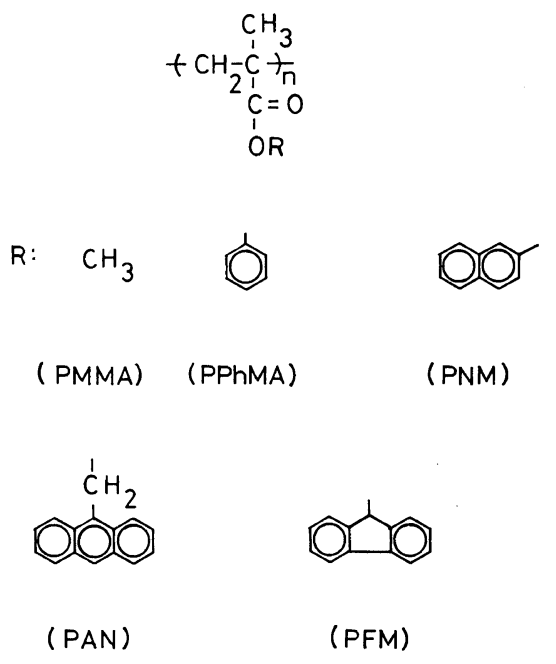

Figure 1. Chemical structures of polymethacrylates examined. PMMA, poly(methyl methacrylate); PPhMA, poly(phenyl methacrylate); PNM, poly( $\beta$-naphthyl methacrylate); PAN, poly(anthrylmethyl methacrylate); PFM, poly(fluorenyl methacrylate). are actually used as resists, and polymer synthesis are relatively easy.

Table I shows the molecular parameters of polymers examined. Etching rates of these polymers against $\mathrm{CF}_{4}$ reactive ion etching (RIE) are also shown in Table I. Clearly absorption edge moves to longer wavelength

Table I. Polymer characteristics and dry-etching rates ${ }^{a}$

\begin{tabular}{lccccc}
\hline Polymer & $\begin{array}{c}M_{w} \\
\left(\times 10^{4}\right)\end{array}$ & $M_{w} / M_{n}$ & $\begin{array}{c}\text { DE } \\
\text { rate }\end{array}$ & $\begin{array}{c}\text { Abs. } \\
\text { edge }\end{array}$ & Sensitivity \\
\hline PMMA & 37 & 2.8 & 730 & 275 & 10 \\
PPhMA & 20 & 2.0 & 340 & 310 & 50 \\
PNM & 8.3 & 3.4 & 260 & 340 & 200 \\
PFM & 3.0 & 2.9 & 340 & 330 & 65 \\
PAN & 7.1 & 3.3 & 230 & 420 & 550 \\
PVK & 90 & 4.9 & 220 & 390 & Negative \\
\hline
\end{tabular}

a $\mathrm{DE}$ rate, dry-etching rate $\left(\mathrm{A} \mathrm{min}^{-1}\right)$; etchant gas, $\mathrm{CF}_{4}$ and $5 \% \mathrm{O}_{2} ;$ Abs. edge, absorption edge (nm). Sensitivity was defined as minimum dose $\left(\mu \mathrm{Ci} \mathrm{cm}^{-2}\right)$ giving zero thickness after development. Developer, monochlorobenzene; PMMA, poly(methyl methacrylate); PPhMA, poly(phenyl methacrylate); PNM, poly( $\beta$-naphthyl methacrylate); PFM, poly(fluorenyl methacrylate); PAN, poly(anthracenemethyl methacrylate); PVK, poly(vinylcabazole); $M_{w}$, weight average molecular weight; $M_{n}$, number average molecular weight.

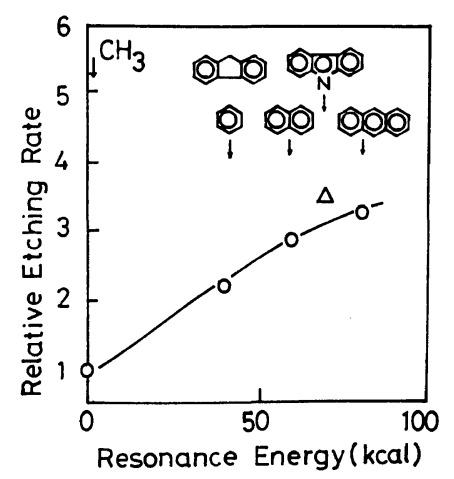

Figure 2. Relationship between the relative dryetching rates and resonance energies. $\bigcirc$ and $\triangle$ indicate polymethacrylates and polyvinylcabazole respectively. The dry etching rate of polymers were investigated by means of reactive ion etching with a parallel plate etching equipment using $\mathrm{CF}_{4}$ containing $5 \%$ oxygen gas. Relative dry-etching rate; etching rate of poly(methyl methacrylate)/etching rate of aromatic polymer. 
and dry-etching durability becomes higher in polymers with larger rings as shown in Table I. Relatively low dry-etching durability of poly(fluorenyl methacrylate) is due to the lack of delocalization between two benzene rings. Figure 2 plots the relationship between the dry-etching rates and resonance energies which are the measure of protective effect of aromatic ring in the side chain. Good correlation indicates the contribution of aromaticity of ring structure to stability against dry-etching reaction.

However, it becomes more difficult to find good solvents for polymers with larger rings, and particularly poly(anthrylmethyl methacrylate) shows a poor solubility. The low solubility restricts the sensitization by chemical modification of polymer and finding a good developer. The resist characteristics of polymers by electron beam exposure were measured and the results are also summarized in Table I. Since the methacrylic main chain degrades by a high energy beam exposure, positive resist features were observed, and the sensitivity was very low in polymers with larger rings.

Among these polymers $\operatorname{poly}(\beta$-naphthyl methacrylate) (PNM) was selected to design new resist, because PNM showed a reasonable solubility and naphthalene rings had an intrinsic absorption up to $340 \mathrm{~nm}$. Furthermore, as the triplet energy level of naphthalene ring is particularly low (62 $\left.\mathrm{kcal} \mathrm{mol}^{-1}\right)$, suitable sensitizers can produce excited states in PNM based polymers by longer wavelength light via triplet-triplet energy transfer. In a previous paper $^{3}$ it was clarified that halogen groups could add a high sensitivity as negative resist to styrene-based polymers with a high contrast, and chloromethyl group showed the highest sensitization. Thus, chloromethyl groups were introduced into PNM. To compare the resist performance of chloromethylated PNMs (CMN), the copolymers of $\beta$ naphthyl methacrylate and glycidyl methacrylate which is known as a representative reactive monomer unit for negative type resist polymers ${ }^{8}$ were synthesized.

\section{EXPERIMENTAL}

\section{Polymer Preparation and Characterization}

Polyaromatic methacrylate monomers were synthesized by the reaction of methacrylic acid and polyaromatic alcohol with cyclohexylcarbodiimide as a dehydration agent. ${ }^{9}$ Poly(methacrylates) except for poly(methyl methacrylate) (PMMA) were prepared by azobisisobutyronitrile (AIBN)-initiated radical polymerization of corresponding methacrylate monomer in toluene at $60^{\circ} \mathrm{C}$ in a sealed tube for $12 \mathrm{~h}$. The polymers were isolated by pouring the reaction solution into methanol, washed with methanol repeatedly and purified by reprecipitation with tetrahydrofuran (THF)-methanol system.

CMN were produced by the chloromethylation of $\operatorname{poly}(\beta$-naphthyl methacrylate). The chloromethylation was carried out in chloromethyl methyl ether with $\mathrm{SnCl}_{4}$ as a catalyst. Copolymers of $\beta$-naphthyl methacrylate and glycidylmethacrylate (NGC) with various monomer feed ratios were prepared by radical polymerization and purified in the same procedure as poly(methacrylates).

PMMA used was Elvacite 2041 available from du Pont. Poly(vinylcarbazole) was obtained from Anan Koryo Co., Ltd.

Molecular weight parameters $\left(M_{w}\right.$ and $\left.M_{n}\right)$ were determined using gel permeation chromatography with a Toyo Soda model HLC802UR GPC. THF was used as the eluting solvent. Chloromethylation ratio of $\mathrm{CMN}$ was calculated on the basis of chlorine content in the elemental analysis of polymer. Glycidyl methacrylate (GMA) content of NGC was determined by measuring the absorption at $320 \mathrm{~nm}$ due to naphthalene ring in the polymer.

\section{Resist Working}

Electron Beam Exposure. Polymers were dis- 
solved in monochlorobenzene and then spincoated onto a silicon wafer with $\mathrm{SiO}_{2}$ to a thickness of $0.5-0.8 \mu \mathrm{m}$. Prebaking and postbaking were carried out under a nitrogen atmosphere, taking $T_{\mathrm{g}}$ of polymers into account. The resist characteristics to electron beam were examined with a computercontrolled electron beam exposure machine ELS-5000 (Elionix). The electron energy used was $20 \mathrm{kV}$. The exposed film was developed by dipping a sample in a suitable developer. THF-2-propanol (2PA) mixed solution was used as a developer for CMN. After development, the resist film was rinsed by dipping the sample in 2PA. The resist thickness before and after the development was measured with Talystep (Rank Taylor Hobson). The electron beam sensitivities were expressed as $D_{0.5}$ and $D_{0}$ which were defined as a dose to leave a half of initial resist thickness and a minimum dose required to form an insoluble gel, respectively. The product of $D_{0}$ and $M_{w}$ was used as the measure of polymer reactivity to eliminate the effect of molecular weight. ${ }^{3}$ Contrast $(\gamma)$ was determined as the slope of the extrapolated linear portion of the dose-thickness curve. ${ }^{10}$

Dry Etching Rate. The dry etching rates of polymers were investigated by means of reactive ion etching (RIE) with a parallel plate etching equipment DEM-451 (ANELVA). $\mathrm{CF}_{4}$ containing $5 \%$ oxygen gas and $\mathrm{CCl}_{4}$ gas were used as etchant gases under the conditions of $60 \mathrm{~m}$ Torr, $150 \mathrm{~W}$ and $10 \mathrm{~m}$ Torr, $100 \mathrm{~W}$ respectively.

$U V$ Exposure. The sensitivity of resist which was coated onto quartz plate to UV rays was examined with a gray scale method using UV rays exposure machine (Orc) equipped with $3 \mathrm{~kW}$ super high pressure mercury lamp. The influence of atmosphere on the polymer sensitivity was evaluated by introducing air into the vacuum chamber of the exposure system. The product of $D_{0}$ and $M_{w}$ was used as the measure of polymer reactivity. Contact printing was carried out using Cobilt Model 2020 photoaligner to replicate the mask patterns.

\section{RESULTS AND DISCUSSION}

\section{Exposure Characteristics to Electron Beam}

Sensitivity and Reactivity. Table II summarizes $M_{w}, M_{w} / M_{n}$, reactive group content, electron beam sensitivity, and contrast $(\gamma)$ for CMN and NGC. Both polymers contain naphthalene ring having different reactive groups as shown in Figure 3.

Though PNM which was a starting polymer of $\mathrm{CMN}$ was positive type, a small degree of chloromethylation changed $\mathrm{CMN}$ from positive to negative type resist. CMN-1 with a molecular weight of $1.4 \times 10^{5}$ had the sensitivity of $4.5 \mu \mathrm{Ccm}^{-2}$ as $D_{0.5}$ and $\gamma$ of 2.5 , which indicated the utility of $\mathrm{CMN}$ in practical electron beam lithography. NGC were also negative type, but their sensitivity was much lower than that of the homopolymer of glyci-

Table II. Polymer and resist characteristics for CMN and $\mathrm{NGC}^{\mathrm{a}}$

\begin{tabular}{lrrrrr}
\hline & & \multicolumn{4}{c}{ Sensitivity, } \\
Polymer & $\begin{array}{c}M_{w} \\
\left(\times 10^{4}\right)\end{array}$ & $M_{w} / M_{n}$ Content & \multicolumn{1}{c}{$D_{0.5}$} & $\gamma$-Value \\
\cline { 3 - 5 } & & & & $\mu \mathrm{C} \mathrm{cm}^{-2}$ \\
PNM & 8.3 & 3.4 & 0 & positive \\
CMN-1 & 14.4 & 5.5 & 0.25 & 4.5 & 2.5 \\
CMN-2 & 10.9 & 4.4 & 0.17 & 4.5 & 2.3 \\
CMN-3 & 10.0 & 5.1 & 0.34 & 3.7 & 2.8 \\
CMN-4 & 9.5 & 3.8 & 0.18 & 5.8 & 1.9 \\
CMN-5 & 9.0 & 3.4 & 0.42 & 3.8 & 2.6 \\
CMN-6 & 9.0 & 3.6 & 0.05 & 11.0 & 2.2 \\
CMN-7 & 8.1 & 4.5 & 0.62 & 5.8 & 2.6 \\
CMN-8 & 6.1 & 3.8 & 0.86 & 4.0 & 2.3 \\
CMN-9 & 2.8 & 1.9 & 0.20 & 13 & 2.8 \\
NGC-1 & 5.4 & 3.6 & 0.05 & 520 & 1.0 \\
NGC-2 & 6.1 & 3.9 & 0.18 & 168 & 1.6 \\
NGC-3 & 3.0 & 2.7 & 0.36 & 108 & 1.7 \\
NGC-4 & 5.7 & 3.2 & 0.55 & 28 & 2.4 \\
NGC-5 & 5.3 & 3.5 & 0.76 & 16 & 1.8 \\
PGMA & 9.6 & 1.9 & 1.0 & 0.52 & 2.0 \\
\hline
\end{tabular}

${ }^{\text {a }} M_{w}$, weight-average molecular weight; $M_{n}$, numberaverage molecular weight. Content, reactive group content. PNM, poly(naphthyl methacrylate); CMN, chloromethylated poly(naphthyl methacrylate); NGC, copolymer of naphthyl methacrylate and glycidyl methacrylate; PGMA, poly(glycidyl methacrylate). 


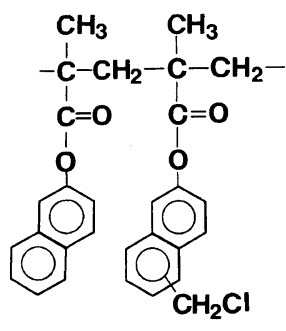

Chloromethylated poly-

NAPHTHYLMETHACRYLATE

CMN<smiles>CC(C)(CC(C)(C)C(=O)Oc1ccc2ccccc2c1)C(=O)OCC1CO1</smiles>

NAPHTHYLMETHACRYLATEGLYCIDYLMETHACRYLATE COPOLYMER NGC

Figure 3. Chemical structures of $\mathrm{CMN}$ and NGC.

dyl methacrylate (PGMA).

The sensitivity of negative resist depends on molecular weight and reactive group content. In any resists in which crosslinking of polymer chains is involved in the resist reaction, the sensitivity is almost proportional to initial $M_{w}$ of polymer. ${ }^{11}$ However, the dependence of sensitivity on the reactive group content is varied according to the kind of reactive group. Figure 4 shows the reactivity expressed as $D_{0} \times M_{w}$ as a function of reactive group content for CMN and NGC. The reactivity of CMN abruptly jumped by a small degree of chloromethylation and increased gradually with chloromethylation ratio. Above $40 \%$ of chloromethylation ratio, further chloromethylation had no effect in the enhancement of polymr reactivity and $\mathrm{CMN}$ followed the relation $D_{0} \times M_{w}=$ constant. By contrast, the epoxy group in NGC increased the reactivity linearly with its content. These tendencies were already observed in many other negative resists. ${ }^{12,13}$ That is, epoxy or vinyl group which is believed to crosslink by chain reaction ${ }^{14}$ exhibited a linear relation between sensitivity and reactive group content, and halogen groups which lack unsaturated bonds tended to show the sensitivity saturation with reactive group content. Normally a very high sensitivity can be obtained by unsaturated group, and moderate sensitivity but high resolution can be realized by halogen groups. However,

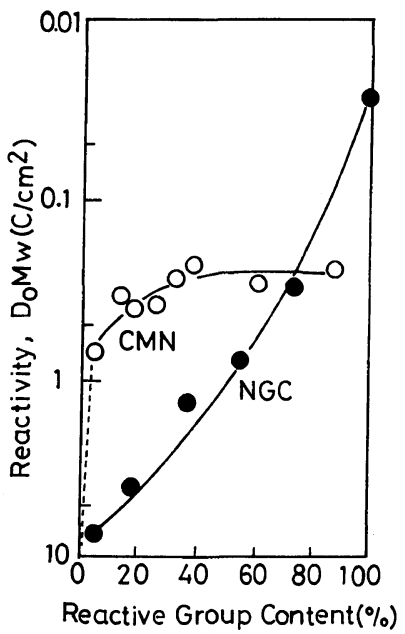

Figure 4. Reactivity expressed as $D_{0} \times M_{w}$ as a function of reactive group content for CMN and NGC.

in the case of PNM, the sensitivity of CMN was higher than that of NGC when compared at same $M_{w}$ and about $80 \%$ reactive group content. This result suggested the protection effect by aromatic rings against the crosslinking by chain reaction was larger than that by stepwise reaction.

Negative electron resists which crosslink by chain reaction such as PGMA suffer from post-irradiation polymerization, ${ }^{2,8}$ which causes non-uniformities in pattern width and thickness in a wafer after development according to the order of electron beam exposure. $\mathrm{CMN}$ showed little post-irradiation polymeri- 
zation effect in analogy with $\mathrm{CMS}^{2}$ While NGC showed a moderate degree of postirradiation polymerization but the degree was not so large in NGC as compared with PGMA. This confirmed the crosslinking reaction of NGC had a nature of chain reaction.

Contrast and Resolution. Contrasts of CMN were generally high in spite of their broad molecular weight distribution. Figure 5 shows the electron beam sensitivity curves for CMN1 and NGC-5. Though NGC-5 had lower $M_{w}$ and much lower sensitivity than CNM-1, contrast of CMN was higher (2.5) than that of NGC (1.8). The tailing near $D_{0}$ in NGC curve was attributed to a low contrast. A steep curve near $D_{0}$ in CMN suggested that the crosslink-

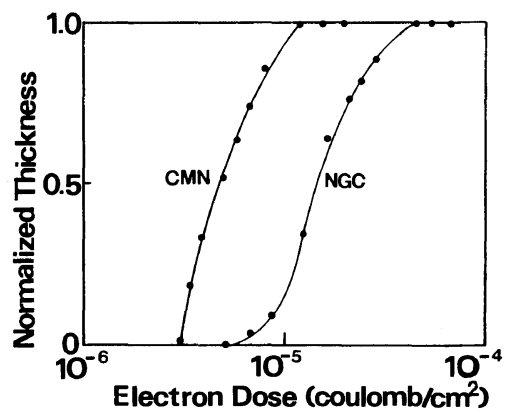

Figure 5. Electron beam sensitivity curves for $\mathrm{CMN}-1$ and NGC-5.

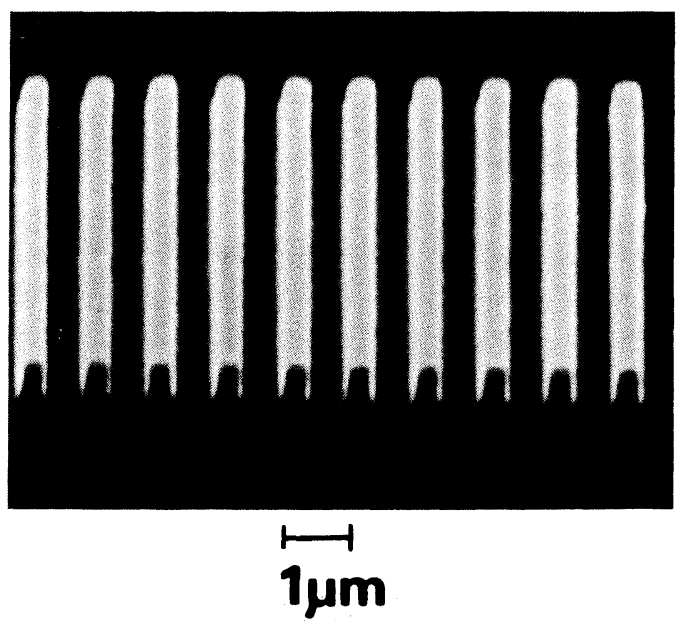

(a) ing of CMN proceeded by a step wise reaction. ${ }^{14}$

As shown in Figure 6, CMN-1 resolved $0.5 \mu \mathrm{m}$ line and space (a), $3 \mu \mathrm{m}$ wide lines with $0.5 \mu \mathrm{m}$ space (b) in an initial thickness of $0.8 \mu \mathrm{m}$. Patterns were developed in THF-2PA (85:15) mixed solution and rinsed in 2-propanol. Pattern profile was sharp in reflection with high $T_{\mathrm{g}}$. Slight rough edge in pattern (b) was attributed to the resist swelling during development. A higher resolution was achieved with decreasing molecular weight of
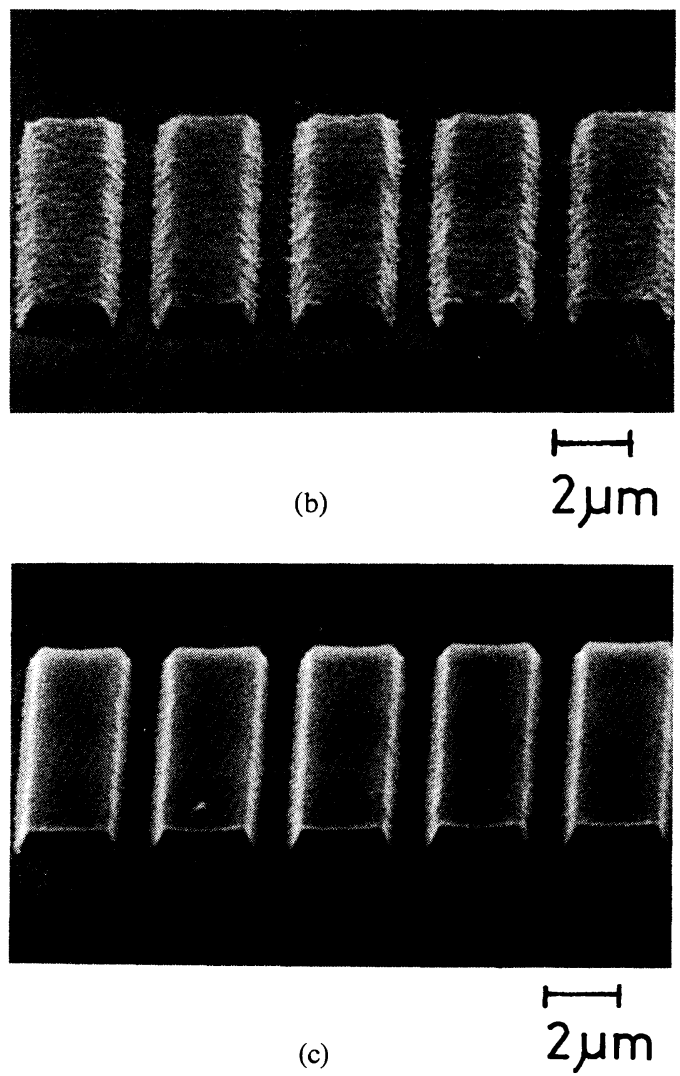

Figure 6. SEM photographs of developed CMN resist patterns after electron beam exposure. $0.5 \mu \mathrm{m}$ line and space (a), $3 \mu \mathrm{m}$ wide lines and $0.5 \mu \mathrm{m}$ space (b) for CMN1 in an initial thickness of $0.8 \mu \mathrm{m}$. Irradiation dose; (a) $8 \times 10^{-6} \mathrm{C} \mathrm{cm}^{-2}$, (b) $6 \times 10^{-6} \mathrm{C} \mathrm{cm}^{-2}$. Patterns were developed in tetrahydrofuran-2-propanol $(85: 15) \mathrm{mix}-$ ed solution and rinsed in 2-propanol. (c) is the same pattern as (b) delineated in CMN-9. Irradiation dose; $1.4 \times 10^{-5} \mathrm{C} \mathrm{cm}^{-2}$. 


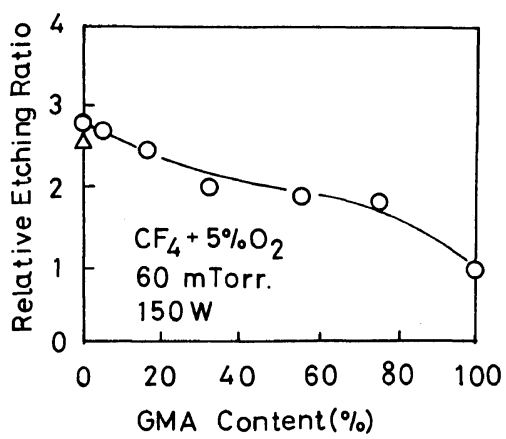

Figure 7. Relationship between glycidylmethacrylate (GMA) content and dry-etching durability for NGC. $\triangle$ indicate chloromethylated poly(naphthyl methacrylate). Etching gas, $\mathrm{CF}_{4}$ containing $5 \%$ oxygen; relative etching rate, etching rate of poly(glycidyl methacrylate)/etching rate of NGC.

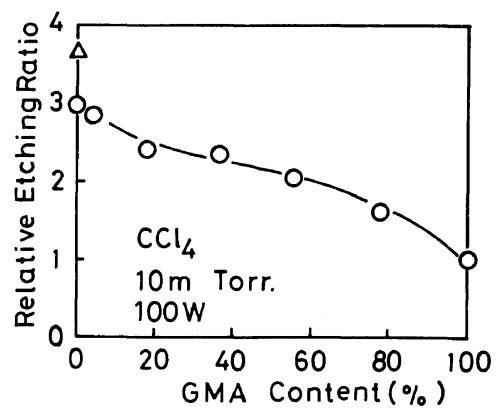

Figure 8. Relationship between GMA content and dry-etching durability against $\mathrm{CCl}_{4}$ gas as etching gas for NGC. $\triangle$ indicate chloromethylated poly(naphthyl methacrylate). The relative etching rate was defined as same as Figure 7.

polymer. Figure 6(c) is the same pattern as (b) delineated in $\mathrm{CMN}-9$.

\section{Dry-Etching Durability}

Figure 7 shows the relation between aromatic unit content and dry-etching durability against $\mathrm{CF}_{4}$ and $5 \% \mathrm{O}_{2}$ mixed gas RIE. Figure 8 shows the same relation against $\mathrm{CCl}_{4}$ gas RIE as Figure 7. Thompson et al. has reported that in the case of glycidyl methacrylate and styrene copolymers, more than $50 \mathrm{~mol} \%$ styrene was necessary to gain practically high dry etching durability. ${ }^{15}$ In NGC for both etching gases, dry-etching durability was gradually improved with content of naphthylmethacrylate unit, and about $30 \%$ of naphthyl methacrylate unit seemed to give enough durability. Eventually, PNM with $100 \%$ of naphthyl methacrylate had about 3 times higher durability than PGMA. Against $\mathrm{CF}_{4}$ RIE the etching rate of $\mathrm{CMN}$, which was little affected by chloromethylation ratio, was equal or somewhat worse than that of PNM. It is noteworthy that $\mathrm{CMN}$ showed higher dry etching durability than PNM against $\mathrm{CCl}_{4}$ gas. Akiya et al. has reported that similar result was found in CMS. ${ }^{16}$ Though this reason is not fully understood, it seems that chlorine in the chloromethyl group participates in dry etching reactions.

As described above, NGC showed lower resolution than $\mathrm{CMN}$ and moderate postirradiation polymerization because corsslinking was carried out by the chain reaction. Furthermore, the increase of naphthyl methacrylate unit was necessary to improve the etching durability, causing the decrease of sensitivity in NGC. These results indicated the utility of introduction of reactive group into aromatic polymer rather than copolymerization of aromatic monomer with aliphatic monomer containing a highly reactive group.

\section{Exposure Characteristics to UV Light}

Sensitivity and Reactivity. Figure 9 shows $\mathrm{UV}$ absorption spectra of CMS, CMN, and CMN containing $3 \mathrm{wt} \%$ Michler's ketone (MK). CMS showed a high sensitivity to deep UV light due to its strong absorption, but had no absorption in mid UV region longer than $300 \mathrm{~nm} .{ }^{17}$ On the other hand, the absorption edge of CMN reached to $340 \mathrm{~nm}$. The crosslinking reaction occurred in $\mathrm{CMN}$ by irradiation of light in this intrinsic absorption region.

In order to sensitize $\mathrm{CMN}$ to longer wavelength light, the sensitivities of $\mathrm{CMN}$ containing various sensitizers were measured, of which results are shown in Table III. Though 


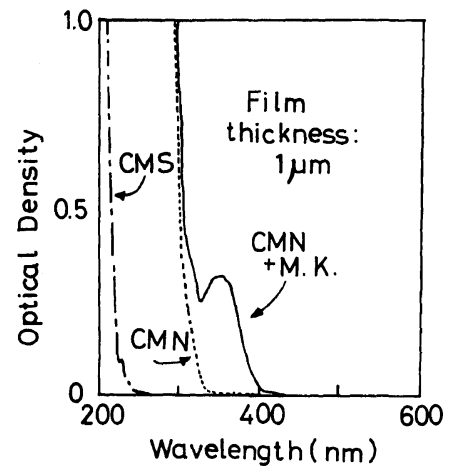

Figure 9. UV absorption spectra of CMS, CMN, and $\mathrm{CMN}$ containing $3 \mathrm{wt}^{\mathrm{o}} \%$ Michler's ketone. The film thickness was $1 \mu \mathrm{m}$.

Table III. Relative sensitivity to UV light for $\mathrm{CMN}$ with photo-sensitizers

\begin{tabular}{|c|c|c|c|}
\hline \multirow{2}{*}{$\begin{array}{c}\text { Sensitizer } \\
\left(3 \mathrm{wt}^{\circ} \%\right)\end{array}$} & \multirow{2}{*}{$\frac{D_{0}}{\mathrm{~mJ} \mathrm{~cm}{ }^{-2}}$} & \multirow{2}{*}{$\begin{array}{c}\text { Relative } \\
\text { sensitivity }\end{array}$} & \multirow{2}{*}{$\frac{\begin{array}{c}\text { Triplet } \\
\text { energy }\end{array}}{\text { kcal mol }^{-1}}$} \\
\hline & & & \\
\hline None & 4151 & 1 & \\
\hline Michler's ketone & 45 & 92 & 62 \\
\hline Nitrofluorenone & 116 & 36 & 59 \\
\hline Nitroacenaphthene & 140 & 30 & 56.6 \\
\hline Anthraquinone & 865 & 4.8 & 62.8 \\
\hline Pyrene & 2770 & 1.5 & 48 \\
\hline Naphtharene & 3110 & 1.3 & 62 \\
\hline Phenanthrene & 2770 & 1.5 & 62 \\
\hline
\end{tabular}

several sensitizers which are known as typical sensitizer, MK showed the maximum sensitization. In the case of CMS, any sensitizers listed in Table III gave no effect. This was because energy level of benzene ring was too low to be sensitized. The triplet energy level of chloromethylated naphthalene rings in CMN was not determined, but this sensitization could be due to effective triplet-triplet energy transfer sensitizer to CMN. The effectiveness of sensitization seemed to be related with the absorption coefficient of sensitizer to light from a super high pressure mercury lamp. As shown in Figure 9, MK was a very suitable sensitizer to $365 \mathrm{~nm}$ light because of its strong absorption. The sensitivity curves of CMN-1

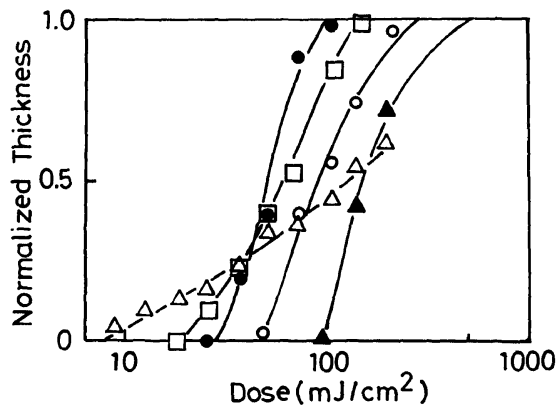

Figure 10. Sensitivity curves of CMN-1 with various content of Michler's ketone. The initial resist thickness was $0.7 \mu \mathrm{m}$. The content of Michler's ketone: $\triangle=$ $24 \mathrm{wt} \% ; \square=12 \mathrm{wt} \% ; \quad=6 \mathrm{wt}^{\mathrm{o}} \% ; \quad \mathrm{O}=3 \mathrm{wt} \% ; \quad \boldsymbol{\Delta}=$ $1 \mathrm{wt} \%$.

with various content of $\mathrm{MK}$ are plotted in Figure 10. The photo-sensitivity, particularly $D_{0}$, was significantly improved by increasing MK content, but above $6 \mathrm{wt} \%$ of MK content the irradiated film did not give enough residual thickness even in a very high dosage region. This could be originated from the strong absorption of MK, which caused absorption non-uniformity in a thickness direction. Thus, an optimum concentration of MK was about $3 \mathrm{wt} \%$, and the sensitivity, $D_{0.5}$, was about 100 $\mathrm{mJ} \mathrm{cm}{ }^{-2}$, which was almost comparable to that of $\mathrm{AZ}$ type photoresists.

CMN had another advantage as negative photoresists, that is, no influence by atmosphere. Figure 11 compares the sensitivity curve of $\mathrm{CMN}-1$ containing $3 \mathrm{wt} \%$ of $\mathrm{MK}$ exposed in air with that exposed in vacuum. Many negative resists showed a considerable decrease in sensitivity upon UV or X-ray exposure in air, and this phenomenon was analyzed by spectroscopic and kinetic studies in terms of oxygen quenching. ${ }^{18,19}$ However, this kind of sensitivity decrease was not observed in poly(vinyl cinnamate) ${ }^{20}$ and CMS. ${ }^{17}$ This can be explained by that the crosslinking reaction of chloromethyl or cinnamate group was very effective and had no character of chain reaction.

Figure 12 shows the chloromethylation ratio dependence of reactivity expressed by $D_{0} \times M_{w}$ 


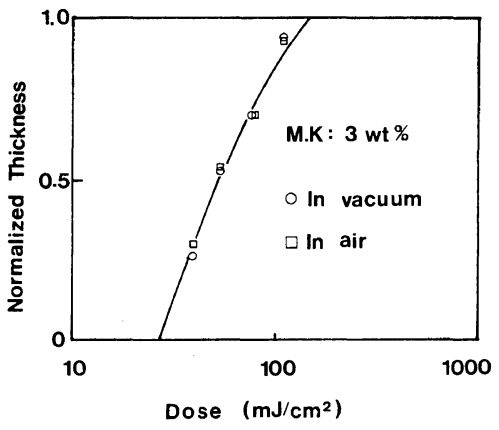

Figure 11. Sensitivity curves of CMN-1 containing $3 \mathrm{wt} \%$ of Michler's ketone exposed in air with that exposed in vacuum. The initial resist thickness was $0.7 \mu \mathrm{m}$.

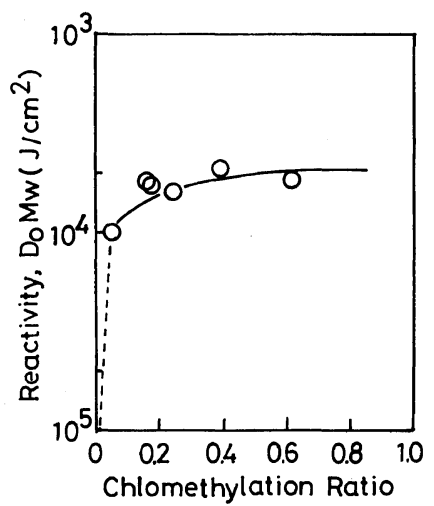

Figure 12. Chloromethylation ratio dependence of reactivity expressed by $D_{0} \times M_{w}$ for CMN with $3 \mathrm{wt} \%$ Michler's ketone.

for $\mathrm{CMN}$ with $3 \mathrm{wt} \% \mathrm{MK}$. This curve is almost identical with that in Figure 4, indicating that photo-crosslinking proceeded via almost the same mechanism as electron beam induced crosslinking reaction.

\section{Pattern Formation by Exposure of Light and Electron Beam}

Figure 13 is an optical microscope image of developed resist patterns with minimum lines of $2 \mu \mathrm{m}$ replicated by contact printing using a photo-aligner. This confirmed that $\mathrm{CMN}$ could be used as photoresist as well as electron beam resist. Figure 14 shows the sensitivity curves to electron beam for $\mathrm{CMN}$ containing

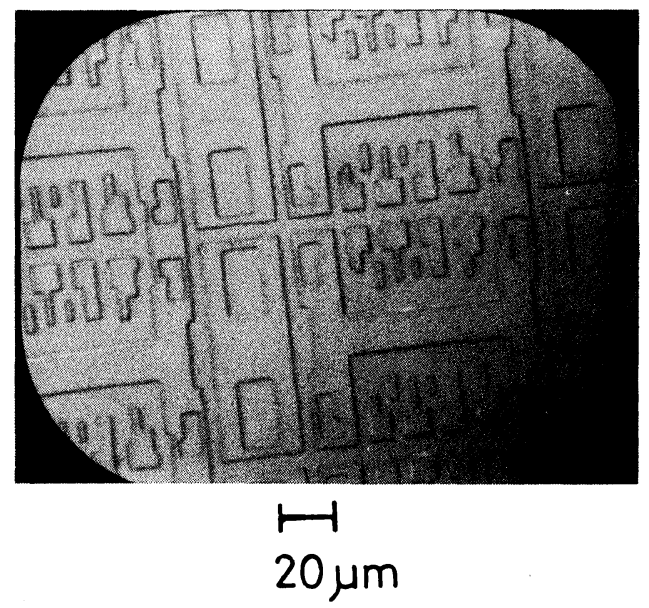

Figure 13. Optical microscope image of developed resist patterns with minimum lines of $2 \mu \mathrm{m}$ replicated by contact printing using photo-aligner. Developing solvent; tetrahydrofuran-2-propanol (85:15), Rinsing solvent; 2-propanol. Irradiation dose; $120 \mathrm{~mJ} \mathrm{~cm}^{-2}$.

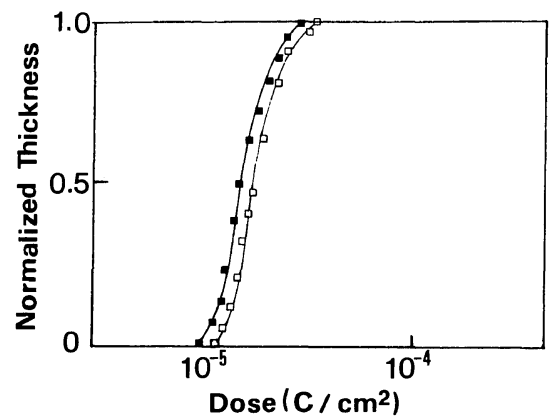

Figure 14. Sensitivity curves to electron beam for CMN (ם) and CMN containing $3 \mathrm{wt} \%$ Michler's ketone ( $\square)$.

$3 \mathrm{wt} \%$ of MK. The electron beam sensitivity was not influenced by the addition of photosensitizers. This suggested the possibility that according to the pattern size, UV and electron beam exposures could be successively carried out before the development. Figure 15 is SEM picture of developed resist pattern after the exposure of both beams, where a very wide pattern $(60 \mu \mathrm{m}$ wide $)$ and fine lines $(1 \mu \mathrm{m}$ wide $)$ were delineated by exposure of UV light and electron beam, respectively. 


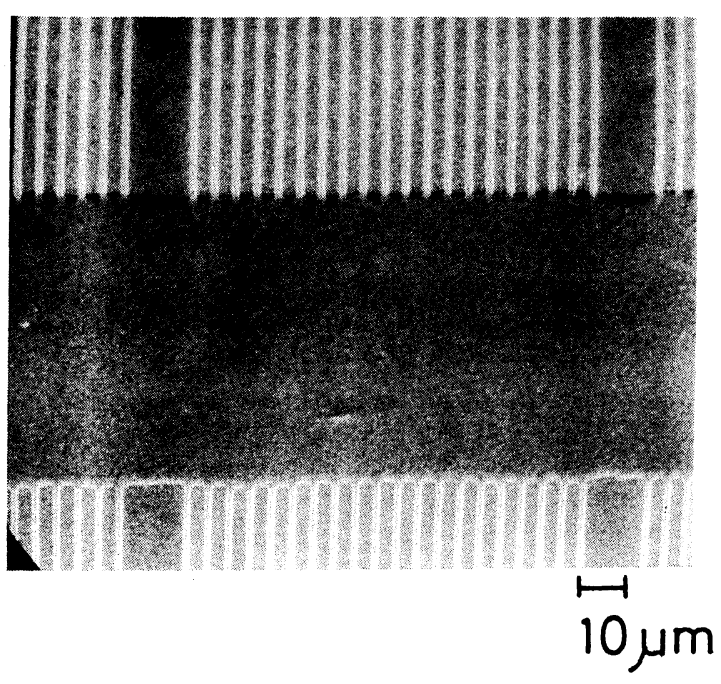

Figure 15. SEM picture of developed resist pattern after the exposure of UV and electron beam. Wide pattern $(60 \mu \mathrm{m}$ wide) and fine lines $(1 \mu \mathrm{m})$ were delineated by the exposure of UV and electron beam respectively. The condition of development was the same as that in Figure 13.

\section{CONCLUSION}

Chloromethylated poly(naphthyl methacrylate)s (CMN) have been evaluated as an electron beam and UV resist. The results are summarized as follows:

(1) CMN showed significantly a high sensitivity and a high resolution to electron beam, though the sensitivity and resolution depended on its molecular weight. For example, the sensitivity for CMN with $M_{w}$ of $1.4 \times 10^{5}$, which resolved $0.5 \mu \mathrm{m}$ line and space was 4.5 $\mu \mathrm{Cm}^{-2}$. In $\mathrm{CMN}$, the high dry etching durability was shown because of high protectivity of naphthalene ring to plasma etching.

(2) CMN could be used as photoresist by the addition of suitable photo-sensitizer. Michler's ketone gave the maximum sensitization to $365 \mathrm{~nm}$ light, and CMN with $3 \mathrm{wt} \%$ Michler's ketone showed a high sensitivity
$\left(D_{0.5}=100 \mathrm{~mJ} \mathrm{~cm}^{-2}\right)$ and resolved $2 \mu \mathrm{m} \mathrm{min-}$ imum line.

Acknowledgements. The authors are indebted to Mr. K. Murase, Dr. S. Sugawara and Mr. K. Harada for their valuable discussions. They thank Miss K. Iimura for her assistance in exposing resists.

\section{REFERENCES}

1. L. F. Thompson, C. G. Wilson, and M. J. Bowden, "Introduction to Microlithography," American Chemical Society, Washington, D.C., 1983.

2. S. Imamura, J. Electrochem. Soc., 126, 1628 (1979).

3. S. Imamura, T. Tamamura, K. Harada, and S. Sugawara, J. Appl. Polym. Sci., 27, 937 (1982).

4. S. Imamura, S. Sugawara, and K. Murase, $J$. Electrochem. Soc., 124, 1139 (1977).

5. J. P. Reekstin and J. H. McCoy, Solid State Technol., 4, 68 (1981).

6. R. D. Moore, Proceedings of the International Conference on Electron and Ion Beam Science and Technology, 1980, p 126.

7. K. Harada, J. Appl. Polym. Sci., 26, 3395 (1981).

8. Y. Taniguchi, Y. Hatano, H. Shiraishi, S. Horigome, S. Nonogaki, and N. Naraoka, Jpn. J. Appl. Phys., 18, 1143 (1979).

9. A. Hassner and V. Alexanian, Tetrahedron Lett., 46, 4475 (1978).

10. L. F. Thompson, Solid State Technol., 27, 000 (1974).

11. H. Y. Ku and L. C. Scala, J. Electrochem. Soc., 116, 980 (1969).

12. L. F. Thompson, L. E. Stillwagon, and E. M. Doerries, J. Vac. Sci. Technol., 15, 938 (1978).

13. S. Nonogaki, H. Morishita, and N. Saitou, Appl. Polym. Symp., 123, 1117 (1974).

14. A. Atoda and H. Kawakatsu, J. Electrochem. Soc., 123, 1519 (1976).

15. L. F. Thompson and E. M. Doerries, J. Electrochem. Soc., 126, 1699 (1979).

16. H. Akiya and T. Kitayama, Proceedings of the 43th Japan Society Applied Physics, 1982, p 213.

17. S. Imamura and S. Sugawara, Jpn. Appl. Phys., 21, 776 (1982).

18. J. M. Moran and G. N. Taylor, J. Vac. Sci. Technol., 16, 2014 (1979).

19. E. Froshe and R. Backhus, Solid State Electron., 14, 95 (1971).

20. R. K. Agnihotri, F. P. Hood, L. G. Lesoine, and J. A. Offenbach, Photogr. Sci. Technol., 15, 938 (1978). 